\title{
BPAN MANIFESTING WITH FEBRILE SEIZURES AND LANGUAGE DELAY:A CASE REPORT FROM BRAZIL
}

\author{
Maria Cecília de Mattos Alves Silva ${ }^{1}$, Thayane Rosas Batista \\ Rezende $^{1}$, Zumira Aparecida Carneiro ${ }^{1}$, Charles Marques Lourenço ${ }^{1,2}$
}

\begin{abstract}
Neurodegeneration with brain iron accumulation (NBIA) is a complex group of hereditary progressive neurodegenerative diseases characterized by deposition of iron in the basal ganglia. Twelve genetic forms of this disorder have been identified in previous studies. Though they have different inheritance mechanisms all are usually associated with abnormal brain MRI findings. One of NBIA types is an X-linked disorder known as Beta-propeller Protein Associated Neurodegeneration (BPAN). Herein we describe the case of a 4-year-old girl with 2 episodes of febrile seizures, a brain MRI showing nonspecific hyperintense signal in the dentate nucleus area, and delays in language and communication development. Her diagnosis was made based on a genetic evaluation where exome sequencing revealed a mutation in the position chrX:48.933.022 region of the WDR45 gene. The literature describes different clinical presentations for BPAN, each with a different prognosis, suggesting a wide range of possible symptoms of BPAN, including mild cognitive delay and even epileptic encephalopathy (EE).
\end{abstract}

Keywords: Febrile seizures; NBIA; BPAN; WDR45 mutation

\section{INTRODUCTION}

Neurodegeneration with brain iron accumulation (NBIA) is a group of hereditary neurodegenerative diseases characterized by iron deposition in the basal ganglia1. NBIA has heterogenous clinical manifestation, but often presents with movement disorders such as parkinsonism and dystonia. Brain magnetic resonance imaging (MRI) of patients with these conditions usually shows abnormal findings. To date, twelve NBI syndromes have been identified, with inheritance patters including autosomal dominant, recessive, and as identified in a recent study, X-linked ${ }^{1-4}$.

Kruer and coworkers (2012) described a novel type of NBIA manifesting as intellectual disability in early childhood ${ }^{1,5}$. The clinical condition remains stable in the second and third decades of life, before entering a phase of progressive neurological and cognitive decline ${ }^{1,5,6}$. Brain MRI findings associates with this syndrome include iron accumulation specifically in the substantia nigra, in addition to global cerebral atrophy ${ }^{1,7}$.

Due to its clinical course, this disease was originally named Static Encephalopathy of childhood with Neurodegeneration in Adulthood (SENDA) 1 . Further studies showed that the condition was caused by a heterozygous mutation in the Xp11.23 region of the WDR45 gene ${ }^{1}$. Since this gene encodes a protein associated with autophagy, the name of the condition was changed to Beta-propeller Protein Associated Neurodegeneration (BPAN) ${ }^{1,3,8}$.

Herein we report the case of a female Brazilian patient harboring a mutation in the WDR45 gene whose first clinical manifestations were febrile seizures and language delay.

Clin Biomed Res. 2021;41(1):91-93

1 Faculdade de Medicina, Centro Universitário Estácio de Ribeirão Preto. Ribeirão Preto, SP, Brasil.

2 Centro Paulista de Diagnóstico, Pesquisa e Treinamento (CPDP), Ribeirão Preto, SP, Brasil.

Corresponding author: Charles Marques Lourenço charlesgenetica@gmail.com Faculdade de Medicina, Centro Universitário Estácio de Ribeirão Preto: Rua Abrahão Issa Halach, 980. 14096-160, Ribeirão Preto, SP, Brasil. 


\section{CASE REPORT}

The patient was a 4-year-old female, born to healthy and non-consanguineous parents, presenting language delay.

She was born by cesarean delivery after an uneventful pregnancy, with normal anthropometric measurements at birth. Developmental milestones were reported to be normal although language delay was present (spoke her first words at 7 months, with no further improvements).

The patient presented two episodes of febrile seizures: at 17 months and at 2 years and 2 months. After her second febrile seizure, a brain MRI showed nonspecific hyperintensity in the dentate nucleus area (Figure 1). Language delay was later confirmed by a speech therapist and misdiagnosed as speech apraxia.

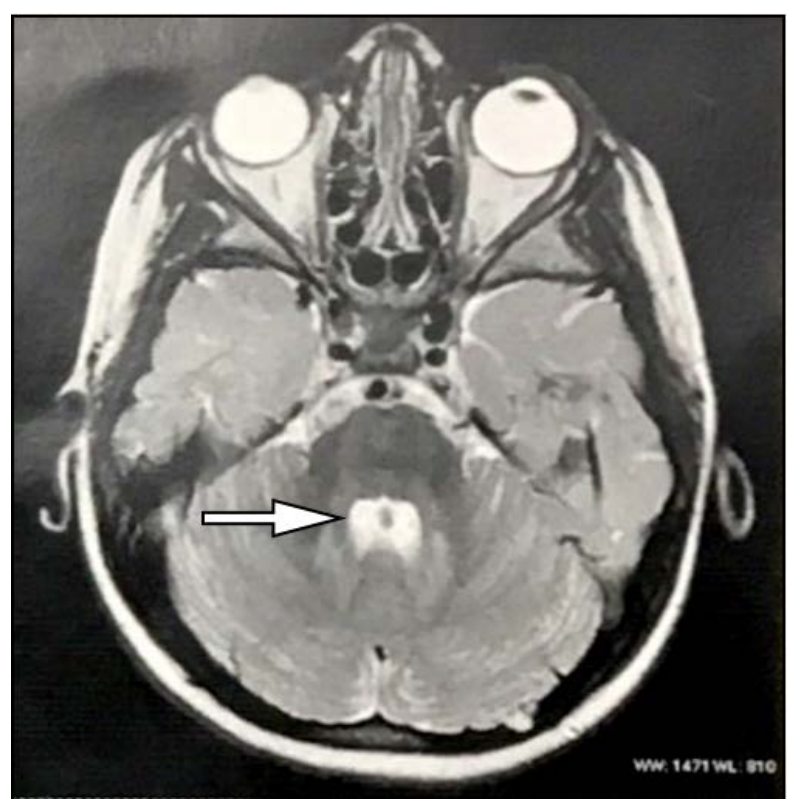

Figure 1: Observe nonspecific hypersignal in the dentate nucleus area on patient's Brain MRI (white arrow).

The patient underwent comprehensive clinical assessments, but no specific diagnosis was established. The possibility of an autism spectrum disorder was considered, but ultimately discarded due to preservation of social skills and lack of typical autism symptoms.

Subsequently, the patient was referred for genetic evaluation. Whole exome sequencing (WES) identified an intronic mutation in the WDR45 gene (c. $830+1 \mathrm{G}>\mathrm{A}$ ) confirming the diagnosis of NBIA type 5 , an X-linked NBIA subtype of the disorder.

The patient has since received rehabilitation treatment, but her language is limited to simple words: yes, no, "mama" (mother) and "vovó" (grandmother). She is currently enrolled in regular school, but is not at the same academic level as her classmates and has shown no improvement in the language. She has no motor deficits, and has shown no clinical deterioration and no further seizures.

\section{DISCUSSION}

According to the literature, the presentation of BPAN is characterized by developmental delayed, especially in language skills, followed by a period of stability and then a progressive decline with symptoms of neurodegeneration ${ }^{1-15}$. Many of the patients do not have an early MRI, as was observed in the present case, possibly because they are not suspected of having metabolic or genetic disorders.

In this case report, the patient's first MRI occurred after her second episode of febrile seizure. The investigation started due to the abnormal finding associated to her speech underdevelopment. Autism spectrum disorder was considered but soon discarded since the patient engaged in social interaction and did not meet other the clinical criteria for this condition. She is now under clinical observation, with no signs of neurological deterioration. Previous studies suggest that patients harboring mutations in the WDR45 gene usually show global neurological decline after the second decade of life ${ }^{11}$.

There is no accurate epidemiological data on NBIA syndromes ${ }^{5}$. Gregory and colleagues (2004) estimated a population prevalence of 1-3/1.000.000, assuming low rates of misdiagnosis and missed cases. BPAN would account for an estimated $1 \%$ to $2 \%$ of these cases ${ }^{1,12}$.

In 2016, Yuri A Zarate et al. reported a familial case of BPAN, supporting the existence of inherited variants and hemizygous germline variants which are non-lethal in males ${ }^{13}$. They described a 20 -year-old boy with MRI's abnormalities and deletions in the WDR45 gene. His mother, sister and father were also tested, and the gene was present in the women. The boy was more severely affected than his relatives, presenting with epilepsy, global developmental delay and totally dependence ${ }^{12}$. Therefore, even though in our case the parents had no genetic mutations, it is important for parents to be tested to detect gonadal and germline mosaicism. A healthy woman might carry the gene and be more likely to pass on the mutation to her male children, resulting in a more severe debilitating condition.

The present findings can also be compared to those of Kyoto Takano et al., who described a 4-year-old male patient. The boy had remarkable developmental delay, non-syndromic epileptic encephalopathy (EE), and MRI evidence of a mild brain atrophy. He was born at 40 weeks and 1 day after an uneventful pregnancy, to unrelated parents with no family history of neurodevelopment disorders. His developmental delay was evident from an early age, but no abnormalities were observed on 
initial MRI scans. Similar to our patient, he developed seizures at 14 months; however, these were not accompanied by fever, and were refractory to multiple antiepileptic drugs. The boy eventually showed global neurological deterioration, and was diagnosed with $\mathrm{EE}$, a group of disorders characterized by intractable seizures that lead to sever cognitive deterioration. Moreover, he showed a de novo intronic hemizygous mutation in the WDR45 gene, identical to our patient, but with more severe neurological impairment ${ }^{14}$.

While many patients have been described and diagnosed in the adulthood, Michelle Long et al. reported on an 18-year-old girl with a WDR45 mutation (c.251A > G, p.Asp84Gly) and pathognomonic MRI findings, but only mild cognitive delay and complete independency ${ }^{15}$. In other words, mutations in the WDR45 gene can result in different phenotypes.
These data demonstrate that neurodegenerative diseases can vary in terms of severity and clinical presentation at any age. It is therefore reasonable to suggest that children like our patient should have regular clinical monitoring to better predict the onset of the severe decline stage and verify whether early intervention can be effective.

Based on our case report and literature review, we suggest that BPAN be included as a differential diagnosis for neurological disorders in children with development delay who present with febrile seizures, or even seizures unaccompanied by fever.

\section{Conflicts of Interest}

The authors declare no conflicts of interest.

\section{REFERENCES}

1. Verhoeven WM, Egger JI, Koolen DA, Yntema H, Olgiati S, Breedveld GJ, et al. Beta-propeller protein-associated neurodegeneration (BPAN), a rare form of NBIA: novel mutations and neuropsychiatric phenotype in three adult patients. Parkinsonism Relat Disodr. 2014;20(3):332-6.

2. Di Meo I, Tiranti V. Classification and molecular pathogenesis of NBIA syndromes. Euro J Paediatr Neurol. 2018;22(2):272-84.

3. Hermann A, Kitzler HH, Pollack T, Biskup S, Krüger S, Funke C, et al. A case of beta-propeller proteinassociated neurodegeneration due to a heterozygous deletion of WDR45. Tremor Other Hyperkinet Mov (N Y). 2017;7:465.

4. Haack TB, Hogarth P, Gregory A, Prokisch $\mathrm{H}$, Hayflick SJ. BPAN: the only X-linked dominant NBIA disorder. Int Rev Neurobiol. 2013;110:85-90.

5. Kruer MC, Boddaert N, Schneuder SA, Houlden HH, Bhatia KP, Gregory $A$, et al. Neuroimaging features of neurodegeneration with brain iron accumulation. AJNR Am J Neuroradiol. 2012;33(3):407-14.
6. Hattingen E, Handke N, Cremer K, Hoffjan S, Kukuk GM. Clinical and imaging presentation of a patient with beta-propeller protein-associated neurodegeneration, a rare and sporadic form of neurodegeneration with brain iron accumulation (NBIA). Clin Neuroradiol. 2017;27(4):481-3.

7. Salomão RP, Pedroso JL, Gama MT, Dutra LA, Maciel RH, GodeiroJunior C, et al. A diagnostic approach for neurodegeneration with brain iron accumulation: clinical features, genetics and brain imaging. Arq Neuropsiquiatr. 2016;74(7):587-96.

8. Kiriyama $\mathrm{Y}$, Nochi $\mathrm{H}$. The function of autophagy in neurodegenerative diseases. Int J Mol Sci. 2015;16(11):26797-812.

9. Van Goethem G, Livingston JH, Warren D, Oojageer AJ, Rice GI, Crow YJ. Basal ganglia calcification in a patient with beta-propeller proteinassociated neurodegeneration. Pediatr Neurol. 2014;51(6):843-45.

10. Schneider SA, Bhatia KP. Syndromes of neurodegeneration with brain iron accumulation. Semin Pediatr Neurol. 2012;19(2):57-66.
11. Tschentscher A, Dekomien G, Ross S, Cremer K, Kukuk GM, Epplen JT, et al. Analysis of the C19orf12 and WDR45 genes in patients with neurodegeneration with brain iron accumulation. $J$ Neurol Sci. 2015;349(1-2):105-9.

12. Gregory AM, Hayflick SJ. Neurodegeneration with brain iron accumulation. Portland: Orphanet Encyclopedia; 2004 [cited 2021 Feb 10]. Available from: https://www.orpha.net/ data/patho/GB/uk-NBIA.pdf

13. Zarate YA, Jones JR, Jones MA, Millan F, Juusola J, Vertino-Bell A, et al. Lessons from a pair of siblings with BPAN. Eur J Hum Genet. 2016;24(7):1080-3.

14. Takano K, Goto K, Motobayashi M, Wakui K, Kawamura R, Yamaguchi T, et al. Early manifestations of epileptic encephalopathy, brain atrophy, and elevation of serum neuron specific enolase in a boy with beta-propeller protein-associated neurodegeneration. Eur J Med Genet. 2017;60(10):521-6.

15. Long M, Abdeen N, Geraghty MT, Hogarth P, Hayflick S, Venkateswaran S. Novel WDR45 mutation and pathognomonic BPAN imaging in a young female with mild cognitive delay. Pediatrics. 2015;136(3):e714-7. 